\section{Correspondence on Rajyaguru et al}

To the EDitor: In their recent article in Journal of Clinical Oncology, Rajyaguru et $\mathrm{al}^{1}$ report on a retrospective comparison of two local ablative methods (stereotactic body radiotherapy [SBRT] and radiofrequency ablation [RFA]) for the management of localized hepatocellular carcinoma (HCC) using the National Cancer Database. The authors find that overall survival is better in the RFA than in the SBRT cohort, even when some parameters are matched. For the comparison of effectiveness of local ablative methods, prospective randomized studies are still missing, and hence, retrospective analyses of large databases such as the study by Rajyaguru et al are relevant as the best evidence at hand. This study is specifically impressive because it reports data on almost 4,000 patients. However, although some limitations are discussed by the authors, several major limitations remain unaddressed, which we would like to highlight.

First, the major weakness of the study design is that information on the liver function of patients is missing (ie, ChildTurcotte-Pugh [CTP] score, which is the predominant prognostic factor, because a majority of patients die as a result of liver failure and portal hypertension). Moreover, data on Barcelona Clinic Liver Cancer stage and presence of portal vein invasion are also missing. The authors attempt to compensate for missing CTP score by providing the Ishak fibrosis score, although this information is available for few patients (SBRT, 16\%; RFA, 29\%). However, the prognostic value of the Ishak score is low compared with that of CTP score, ${ }^{2}$ and comorbidity scores also cannot compensate for missing CTP score. Finally, clinical TNM stage I and II data are assumed to represent exclusively $\mathrm{T}$ stage, ignoring $\mathrm{N}$ stage, and not to represent prognostic stage groups I or II. Therefore, the authors' matched pair analysis explores only minor prognostic variables and thus cannot achieve properly matched patient cohorts. We suspect that the difference in survival reported is a result of selection bias, mainly resulting from imbalanced CTP scores and Barcelona Clinic Liver Cancer stages.

Second, this analysis excludes patients receiving any form of adjuvant or neoadjuvant therapy or other forms of local ablative therapies. This is intriguing, because the typical course of the disease almost inadvertently requires further treatment, as discussed by the authors. This exclusion raises important uncertainties about patient selection, because it does not reflect common practice.

Third, regarding patient numbers, there is a major imbalance between the two treatment arms (SBRT, $\mathrm{n}=296 v$ RFA, $\mathrm{n}=3,684$ ), which underlines the risk of a major selection bias. One has to assume that only patients who were not candidates for other treatment options were referred for SBRT. Interestingly, patients receiving RFA were more likely to have private insurance, and they were younger compared with patients receiving SBRT. Both of these factors would have a direct impact on survival.

Fourth, much of the authors' discussion focuses on local control (LC) after RFA and SBRT, and interestingly, the authors state that LC in HCC may not translate into improved progressionfree or overall survival. Unfortunately, no data on LC are reported in this analysis to prove this hypothesis, which would, if true, favor neither RFA nor SBRT. However, SBRT and RFA are local ablative therapies, and multiple recent publications conclude that even though SBRT shows an advantage for larger tumors, both techniques result in the same LC rates. ${ }^{3,4}$

Fifth, the data presented originate from the years between 2003 and 2014. SBRT at that time (2003 to 2010) was in the early phase of development and optimization (ie, there was no standardized technique or radiation dose for liver tumors). In contrast, there are now widely accepted guidelines ${ }^{5}$ and reports that advanced technologies for liver SBRT and the use of sufficiently high radiation doses are significantly associated with improved outcomes, perhaps even exceeding those achievable with RFA, ${ }^{3,6}$ with low toxicity ${ }^{7}$ and good quality of life. ${ }^{8}$ Proper knowledge of SBRT details regarding prescription dose, motion management, and image guidance as well as their correlation with LC is therefore of paramount importance in interpreting these outcome data.

In summary, Rajyaguru et $\mathrm{al}^{1}$ report rather surprising differences in survival between RFA and SBRT. A closer look at their work reveals that a number of highly important prognosis factors for patients with HCC are not taken into consideration in the statistical analysis. Therefore, we would like to emphasize these weaknesses and suggest that physicians treating HCC contribute to prospective randomized trials that compare the two methods.

\section{Judit Boda-Heggemann}

University Medical Center Mannheim, University of Heidelberg, Mannheim, Germany

\section{Eleni Gkika}

University Medical Center Freiburg, Freiburg im Breisgau, Germany

\section{Nicolaus Andratschke}

University Hospital Zürich, Zürich, Switzerland

\section{Oliver Blanck}

University Medical Center Schleswig-Holstein, Kiel, Germany

\section{Matthias Guckenberger}

University Hospital Zürich, Zürich, Switzerland

\section{Frank Lohr}

Azienda Ospedaliero-Universitaria di Modena, Modena, Italy

\section{Thomas B. Brunner}

University Medical Center Magdeburg, Magdeburg, Germany

\section{ACKNOWLEDGMENT}

Written on behalf of the Stereotactic Radiotherapy Working Group of the German Association for Radiation Oncology. J.B.-H. and E.G. contributed equally to this work.

\section{AUTHORS' DISCLOSURES OF POTENTIAL CONFLICTS OF INTEREST}

Disclosures provided by the authors are available with this article at jco.org. 


\section{REFERENCES}

1. Rajyaguru DJ, Borgert $A J$, Smith $A L$, et al: Radiofrequency ablation versus stereotactic body radiotherapy for localized hepatocellular carcinoma in nonsurgically managed patients: Analysis of the National Cancer Database. J Clin Oncol 36:600-608, 2018

2. Shen JY, Li C, Wen TF, et al: A simple prognostic score system predicts the prognosis of solitary large hepatocellular carcinoma following hepatectomy. Medicine (Baltimore) 95:e4296, 2016

3. Wahl DR, Stenmark MH, Tao Y, et al: Outcomes after stereotactic body radiotherapy or radiofrequency ablation for hepatocellular carcinoma. J Clin Oncol 34:452-459, 2016

4. Ohri N, Dawson LA, Krishnan S, et al: Radiotherapy for hepatocellular carcinoma: New indications and directions for future study. J Natl Cancer Inst 108: djw133, 2016
5. Sterzing F, Brunner TB, Ernst I, et al: Stereotactic body radiotherapy for liver tumors: Principles and practical guidelines of the DEGRO Working Group on Stereotactic Radiotherapy. Strahlenther Onkol 190:872-881, 2014

6. Seo YS, Kim MS, Yoo HJ, et al: Radiofrequency ablation versus stereotactic body radiotherapy for small hepatocellular carcinoma: A Markov model-based analysis. Cancer Med 5:3094-3101, 2016

7. Sapir E, Tao Y, Schipper MJ, et al: Stereotactic body radiation therapy as an alternative to transarterial chemoembolization for hepatocellular carcinoma. Int J Radiat Oncol Biol Phys 100:122-130, 2018

8. Mutsaers A, Greenspoon J, Walker-Dilks C, et al: Systematic review of patient reported quality of life following stereotactic ablative radiotherapy for primary and metastatic liver cancer. Radiat Oncol 12:110, 2017

DOI: https://doi.org/10.1200/JCO.2018.78.1542; published at jco.org on June 26, 2018. 


\section{AUTHORS' DISCLOSURES OF POTENTIAL CONFLICTS OF INTEREST}

\section{Correspondence on Rajyaguru et al}

The following represents disclosure information provided by authors of this manuscript. All relationships are considered compensated. Relationships are self-held unless noted. I = Immediate Family Member, Inst = My Institution. Relationships may not relate to the subject matter of this manuscript. For more information about ASCO's conflict of interest policy, please refer to www.asco.org/rwc or ascopubs.org/jco/site/ifc.

\section{Judit Boda-Heggemann}

No relationship to disclose

Eleni Gkika

No relationship to disclose

Nicolaus Andratschke

Honoraria: AstraZeneca

Research Funding: Varian Medical Systems

Travel, Accommodations, Expenses: BrainLAB

\section{Oliver Blanck}

No relationship to disclose

\section{Matthias Guckenberger}

Research Funding: Varian Medical Systems

Frank Lohr

No relationship to disclose

Thomas B. Brunner

No relationship to disclose 